\title{
Coagulation abnormalities in patients with single- ventricle physiology precede the Fontan procedure
}

Kirsten C. Odegard, $\mathrm{MD}^{\mathrm{a}}$

Francis X. McGowan, Jr, MD ${ }^{a, e}$

James A. DiNardo, MDa

Robert A. Castro, MTa

David Zurakowski, $\mathrm{PhD}^{\mathrm{c}}$

Caroline M. Connor, MD

Dolly D. Hansen, MDa

Ellis J. Neufeld, MD, PhDa

Pedro J. del Nido, MD ${ }^{d}$

Peter C. Laussen, MBBS ${ }^{a}$
From the Departments of Anesthesia, Cardiac Surgery, ${ }^{\mathrm{b}}$ Biostatistics, ${ }^{\mathrm{c}}$ and Division of Hematology, ${ }^{\mathrm{d}}$ Children's Hospital, Boston, and Harvard Medical School, ${ }^{\mathrm{e}}$ Boston, Mass.

Supported in part by National Institutes of Health grants HL04184, ${ }^{\mathrm{d}}$ HL52589, and HL66186,a,e and the Children's Hospital Anesthesia Foundation.

Received for publication May 29, 2001; revisions requested Aug 6, 2001; revisions received Aug 27, 2001; accepted for publication Aug 31, 2001.

Address for reprints: Kirsten C. Odegard, MD, Cardiac Anesthesia Service, 300 Longwood Ave, Children's Hospital, Boston, MA 02115 (E-mail: kirsten.odegard@tch. harvard.edu).

J Thorac Cardiovasc Surg 2002;123:459-65

Copyright (C) 2002 by The American Association for Thoracic Surgery

0022-5223/2002\$35.00 + $0 \quad$ 12/1/120010

doi:10.1067/mtc.2002.120010
Objective: Thromboembolic events in patients who have undergone the Fontan operation have been reported to be as high as $20 \%$ to $33 \%$. A hypercoagulable state with deficiencies in proteins $\mathrm{C}$ and $\mathrm{S}$ has been implicated. Using age-matched control subjects, we evaluated whether an altered coagulation state is present earlier in the course of staged single-ventricle repair.

Methods: After informed consent had been obtained, coagulation factors were assayed in 36 infants (mean age, $7.7 \pm 3.6$ months) with single-ventricle cardiac defects immediately before undergoing the bidirectional Glenn procedure; 34 infants (mean age, $8.4 \pm 2.6$ months) without cardiac disease were assayed as control subjects. Concentration of factors II, V, VII, VIII, IX, and X; antithrombin III; plasminogen; proteins $\mathrm{C}$ and $\mathrm{S}$; fibrinogen; serum albumin; and liver enzymes were measured. Normal reference intervals on the basis of the control subjects were determined by using $95 \%$ confidence limits. Patient demographic and hemodynamic variables were evaluated as possible predictors of coagulation abnormalities.

Results: Concentrations of protein C; factors II, V, VII, IX, and X; plasminogen; fibrinogen; and antithrombin III were significantly lower in the pre-Glenn infants compared with in the age-matched control subjects (all $P<.001$, Student $t$ test). On the basis of multiple logistic regression, no specific hemodynamic variables were predictive of a procoagulant or anticoagulant deficiency. Ventricular dysfunction did predict the presence of multiple coagulation abnormalities $(P<.001)$.

Conclusion: Procoagulant and anticoagulant factor abnormalities occur early in the course of single-ventricle repair and precede the cavopulmonary connection.

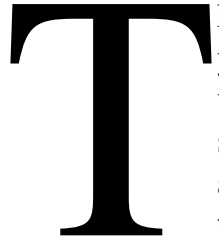

he Fontan operation is currently performed for children older than 1 year with a wide variety of congenital heart defects that preclude a 2ventricle repair. It separates the systemic and pulmonary circulations such that the systemic venous return is directed to the lungs in the absence of a pulmonary ventricle. Since the original Fontan operation, ${ }^{1}$ several modifications have been undertaken that have improved early and late mortality and morbidity. For example, a recent study by Stamm and colleagues $^{2}$ reported long-term follow-up of 220 patients who had undergone the lateral tunnel modification of the Fontan operation for various cardiac malformations. An estimated survival of $93 \%$ at 5 years and $91 \%$ at 10 years was reported. Nevertheless, these patients continue to have numerous abnormalities that compli- 
cate perioperative management and compromise long-term outcome. ${ }^{3}$ Thromboembolic events, arrhythmias, and protein-losing enteropathy are particularly problematic. ${ }^{4}$

Thromboembolism may occur in the immediate postoperative period or later. The incidence of thromboembolic events in patients with Fontan physiology is uncertain but may be as high as $20 \%$ to $33 \%$ overall. ${ }^{5-7}$ Increased venous pressure, hepatic dysfunction, stasis of flow through the right atrial baffle and pulmonary circulation, and increased resting venous tone are some of the factors that may contribute to an increased risk of clot formation in patients with Fontan physiology. Furthermore, abnormal coagulation parameters after the Fontan procedure involving both procoagulant and anticoagulant factors have also been described. ${ }^{8-11}$

These previous studies suggested that the alterations in the coagulation factors found in some patients undergoing the Fontan procedure are a direct consequence of the anatomic and physiologic changes that result from the Fontan operation. However, it is possible that coagulation abnormalities may occur earlier in patients with single-ventricle disease, either because of other physiologic derangements (eg, cyanosis, low cardiac output, and venous congestion) or perhaps because of genetic predisposition. Coagulation status has not been assessed earlier in the course of patients with single-ventricle physiology. A major limitation of the previous studies that strongly implicated Fontan physiology as the primary cause of abnormal clotting parameters is the lack of age-appropriate control subjects. ${ }^{12,13}$ Establishing age-related normal values in infants and children is critical to correctly addressing this question because coagulation factor concentrations and activities mature significantly and at varying rates after birth, with some not approaching adult values until late childhood. ${ }^{12-15}$

We therefore conducted this single-center prospective cohort study to delineate coagulation parameters in infants with single-ventricle physiology before the bidirectional Glenn (BDG) and Fontan procedures. This was done by evaluating procoagulant and anticoagulant factor levels immediately before the BDG procedure and using normal infants of similar age without congenital heart disease as age-matched control subjects.

\section{Methods}

After institutional review board approval and informed parental consent had been obtained, 36 patients between the ages of 3.2 and 19 months with a variety of single-ventricle congenital heart defects were prospectively enrolled immediately before undergoing a BDG procedure. Patients were excluded if they had preexisting hematologic disorders or concurrent coagulopathies or if the patients were receiving warfarin sodium (Coumadin); 23 patients had been receiving aspirin, but this was discontinued 7 to 10 days before the operation.

Blood samples from all patients $(8 \mathrm{~mL})$ were obtained from a central venous catheter or an indwelling arterial catheter at 2 time points: (1) immediately after induction of general anesthesia and (2) 24 hours postoperatively in the intensive care unit (ICU). Measured parameters included hemoglobin level, hematocrit level, platelet count, prothrombin time (PT), and activated partial prothrombin time (APTT). The circulating anticoagulant factors measured were protein $\mathrm{C}$, protein $\mathrm{S}$, plasminogen, and antithrombin III; the procoagulant factors measured were factors II, V, VII, VIII, IX, and $\mathrm{X}$ and fibrinogen.

The Coulter T 660 (Beckman Coulter, Inc, Miami, Fla) automated hematology analyzer was used to measure hemoglobin, hematocrit, and platelet levels. Coefficients of variation within and between assays at levels 1,2 , and 3 were as follows: hemoglobin, $1.8 \%, 1.0 \%$, and $0.9 \%$; hematocrit, $1.8 \%, 1.2 \%$, and $1.5 \%$; and platelets, $4.8 \%, 2.7 \%$, and $1.8 \%$, respectively.

The ACL 3000 Plus (Automated Coagulation Laboratory, Beckman Coulter, Inc, Fullerton, Calif) was used to assay the individual procoagulant and anticoagulant factors, as well as the PT and APTT. The ACL device contains 2 measuring systems: (1) nephelometry, which is used to detect clot formation as the end point, and (2) photometry, which is used for reading chromogenic substrate assays. Blood for these assays was collected in citrated plasma (3.2\% buffered sodium citrate) from an indwelling cannula, from which $10 \mathrm{~mL}$ of blood had been aspirated to remove residual heparin. The blood was immediately centrifuged at 13,000 rpm for 5 minutes, and the plasma layer was removed. The PT, APTT, and fibrinogen levels were measured immediately by means of nephelometry (coefficient of variation, respectively: PT, $1.2 \%$ and $2.3 \%$; APTT, $2.1 \%$ and $4.8 \%$; and fibrinogen, $3.1 \%$ and $5.5 \%$ ). Remaining plasma was stored at $-70^{\circ} \mathrm{C}$ in $200-\mu \mathrm{L}$ aliquots for batch performance of the other coagulation assays, as described below.

Protein $\mathrm{C}$ and $\mathrm{S}$ activity were both measured by using functional clotting assays. Protein $\mathrm{C}$ activity was determined on the basis of the prolongation of the APTT by using the Staclot Protein C kit (Diagnostica Stago, Asnieres-Sur-Seine, France), according to the manufacturer's directions. In this assay, activated protein $\mathrm{C}$ inhibits factor V and VIII activity, thus prolonging the APTT of a system in which all the factors, with the exception of protein $\mathrm{C}$, are present in excess; protein $\mathrm{C}$ is derived from the sample being tested (coefficient of variation, $1.8 \%$ and $2.4 \%$ ).

Protein $\mathrm{S}$ activity was determined on the basis of the principle of factor Va inhibition by using the Staclot Protein $\mathrm{S}$ kit (Diagnostica Stago). The principle of the test is based on the cofactor activity of protein $\mathrm{S}$, which enhances the anticoagulant action of activated protein $\mathrm{C}$. This enhancement is reflected by the prolongation of the clotting time of a system enriched with factor $\mathrm{Va}$ (coefficient of variation, $7.9 \%$ and $3.8 \%$ ).

Extrinsic coagulation factors (factors II, V, VII, and X) were each determined by performing a modified PT assay. Intrinsic coagulation factors (factors VIII and IX) were determined by performing a modified APTT assay. In these assays correction of the clotting time of plasma specifically deficient in the factor being tested is proportional to the concentration (activity percentage) of that factor in the patient plasma interpolated from a calibration curve. Factor-deficient plasma factors II, V, VII, VIII, IX, and $\mathrm{X}$ were obtained from Instrument Laboratory (Lexington, Mass; coefficients of variation, respectively: factor 
II, $2.3 \%$ and $2.7 \%$; factor V, $3.3 \%$ and $2.3 \%$; factor VII, $2.5 \%$ and $2.2 \%$; factor VIII, $4.9 \%$ and $3.7 \%$; factor IX, $3.2 \%$ and $3.1 \%$; and factor $\mathrm{X}, 3.0 \%$ and $2.0 \%$ ).

Antithrombin III activity was determined by using a synthetic chromogenic substrate assay based on factor Xa inactivation (antithrombin III, Instrument Laboratory; coefficient of variation, $4.3 \%$ and $2.8 \%$ ). Plasma plasminogen was activated through reaction with an excess of streptokinase in the presence of fibrinogen. Plasminogen content was then determined on the basis of a synthetic chromogenic substrate assay, according to the manufacturer's directions (Plasminogen, Instrument Laboratory; coefficient of variation, $4.5 \%$ and $3.7 \%$ ).

Because altered hepatic dysfunction can contribute to coagulation factor abnormalities, serum alkaline phosphatase, gammaglutamyl transferase, alanine transaminase, aspartate transaminase, total bilirubin, albumin, and total protein were measured in all patients and compared with normal values for our laboratory.

\section{Age-matched Control Coagulation Parameters}

After informed written parental consent had been obtained, 34 healthy infants ( 8 girls and 26 boys; mean age, $8.4 \pm 2.6$ months; age range, 3-13 months) undergoing minor day surgical procedures were studied. A total of $1.8 \mathrm{~mL}$ of blood was taken from each patient after placement of an intravenous catheter and collected into citrated plasma (3.2\% buffered sodium citrate). Blood samples were immediately centrifuged at $13,000 \mathrm{rpm}$ for $5 \mathrm{~min}$ utes, and the plasma was stored at $-70^{\circ} \mathrm{C}$ for subsequent batch analyses. Proteins $\mathrm{C}$ and $\mathrm{S}$, plasminogen, fibrinogen, antithrombin III, and factors II, V, VII, VIII, IX, and X were analyzed as described above.

\section{Potential Hemodynamic Factors}

As part of the routine preoperative evaluation, all patients underwent elective cardiac catheterization and echocardiography. Ventricular morphology (morphologic right or left systemic ventricle), ventricular function, and atrioventricular valve and semilunar valve function were assessed by means of preoperative echocardiography. Superior vena cava oxygen saturation $\left(\mathrm{SvO}_{2}\right)$, the ratio of pulmonary to systemic blood flow (Qp/Qs), atrial pressure, pulmonary artery pressure, systemic ventricular end-diastolic pressure (EDP), and the presence of systemic ventricle outflow obstruction or aortic arch obstruction were assessed by means of preoperative cardiac catheterization.

\section{Statistical Analysis}

An a priori power analysis revealed that a total sample of 60 patients (30 control and 30 BDG patients) would provide $82 \%$ power to detect an effect size of 1.0 (mean difference/common $\mathrm{SD}$ ) with respect to each of the 11 coagulation factors and protein levels on the basis of a 2-sample Student $t$ test applying a Bonferroni-corrected $\alpha$ level of .005 (ie, 0.05/11). This sample size would also provide a statistical power exceeding $95 \%$ for comparisons between pre- and post-BDG values on the basis of paired $t$ tests (version 4.0, nQuery Advisor; Statistical Solutions, Boston, Mass). All 11 coagulation and protein variables were assessed for normality by the Wilk-Shapiro test, and no significant departures were identified. Normal reference ranges were based on the empirical $95 \%$ confidence intervals and defined according
TABLE 1. Cardiac diagnoses of patients undergoing BDG

\begin{tabular}{lcl}
\hline Diagnosis & No. & \multicolumn{1}{c}{ Prior operation } \\
\hline DORV & 7 & 1 Norwood, 5 BTS, 1 PAB \\
DORV/PS & 2 & None \\
DORV/PA & 1 & BTS \\
DORV/malalignment CAVC & 1 & None \\
HLHS & 8 & 8 Norwood \\
PA/TGA & 2 & 2 BTS \\
TA/NRGV & 5 & 2 BTS/2 Norwood \\
TA/TGA & 1 & PAB \\
TA/PS & 2 & 1 BTS/1 Norwood \\
TA/PA & 1 & BTS \\
DILV & 2 & 1 BTS/1 Norwood \\
DILV/PS & 2 & 1 PPM/1 None \\
TGA/PS & 1 & None \\
TGA/IAA & 1 & Norwood
\end{tabular}

$D O R V$, Double-outlet right ventricle; $P S$, pulmonary stenosis; $P A$, pulmonary atresia; CAVC, common atrioventricular canal defect; $H L H S$, hypoplastic left heart syndrome; TGA, transposition of great arteries; $T A$, tricuspid atresia; NRGV, normal related great vessels; DILV, double-inlet left ventricle; $I A A$, interrupted aortic arch; $B T S$, Blalock-Taussig shunt; $P A B$, pulmonary artery band; $P P M$, permanent pacemaker.

to 2.5 and 97.5 percentiles. ${ }^{16}$ Control values were compared with pre- and post-BDG values by using the 2 -sample Student $t$ test. Changes between pre- and post-BDG values were evaluated with paired $t$ tests. Univariate and multiple stepwise logistic regression was used to determine whether any patient, hemodynamic, or laboratory variables were predictive of a coagulation abnormality. Candidate variables considered in these analyses included sex, weight, prior procedure, ventricle morphology (left or right), EDP, $\mathrm{SvO}_{2}$, Qp/Qs ratio, common atrial pressure, ventricular function, atrioventricular valve regurgitation, and serum albumin levels. The likelihood ratio test was used to determine the significance of predictors. In addition, multiple linear regression was used to determine any predictors of the number of coagulation abnormalities, ranging from 0 to 11 . For comparisons between control patients and pre-BDG and post-BDG patients, a Bonferroni corrected $P<$ .005 was considered significant to account for the 11 coagulation variables being compared. Analysis of the data was performed with the SPSS (version 10.1; SPSS Inc, Chicago, Ill) and SAS (version 6.12; SAS Institute, Cary, NC) software packages. Continuous variables are expressed in terms of means \pm SD. All reported $P$ values are 2 -tailed.

\section{Results}

The mean age for the 36 patients ( 15 girls and 21 boys) was $7.7 \pm 3.6$ months, and the mean weight was $6.3 \pm 1.2 \mathrm{~kg}$. Patient diagnoses and prior procedures are summarized in Table 1; 21 patients had a morphologically right ventricle, and 15 had a morphologically left ventricle. Prior procedures in the patients are also included in Table 1. Pre-BDG hemodynamic variables, as assessed either with echocardiography or cardiac catheterization, are shown in Table 2. 
TABLE 2. Preoperative hemodynamic characteristics

\begin{tabular}{lc}
\hline & No. $(\%)$ \\
\hline Ventricular function & \\
Normal & $25(71 \%)$ \\
Mildly depressed & $8(23 \%)$ \\
Moderately-severely depressed & $2(6 \%)$ \\
AVVR & \\
No regurgitation & $12(34 \%)$ \\
Trivial-mild & $21(60 \%)$ \\
Moderate-severe & $2(6 \%)$ \\
EDP & \\
Mean (mm Hg) & $9.1 \pm 3.9$ \\
$<12 \mathrm{~mm} \mathrm{Hg}$ & $22(69 \%)$ \\
$\geq 12 \mathrm{~mm} \mathrm{Hg}$ & $10(31 \%)$ \\
Atrial pressure & \\
Mean $(\mathrm{mm} \mathrm{Hg})$ & $6.6 \pm 4.2$ \\
$<5 \mathrm{~mm} \mathrm{Hg}$ & $12(36 \%)$ \\
$\geq 5 \mathrm{~mm} \mathrm{Hg}$ & $21(64 \%)$ \\
$\mathrm{PAP}$ & \\
$\mathrm{Mean}(\mathrm{mm} \mathrm{Hg})$ & $16 \pm 5.4$ \\
$<12 \mathrm{~mm} \mathrm{Hg}$ & $5(16 \%)$ \\
$\geq 12 \mathrm{~mm} \mathrm{Hg}$ & $27(84 \%)$ \\
$\mathrm{Qp} / \mathrm{Os}$ & $1.5 \pm 0.9$ \\
$\mathrm{PaO}{ }_{2}(\mathrm{~mm} \mathrm{Hg})$ & $47 \pm 10$ \\
$\mathrm{SvO}_{2}(\%)$ & $50 \pm 10$ \\
$\mathrm{Sp0}{ }_{2}(\%)$ & $80 \pm 10$ \\
\hline $\mathrm{Da}$ &
\end{tabular}

Data are expressed as number (\%) or mean \pm SD as shown. AVVR, Atrioventricular valve regurgitation; $P A P$, pulmonary artery pressure; $\mathrm{SpO}_{2}$, oxygen saturation.

For control subjects, the mean age was $8.4 \pm 2.6$ months. The calculated normal reference ranges for coagulation factors are shown in Table 3. Normal ranges were based on the empirical $95 \%$ confidence intervals derived from the 2.5 and 97.5 percentile confidence limits.

The mean hematocrit, platelet count, PT, APTT, and liver function test results for patients before the BDG procedure are shown in Table 4. In all but one patient, abnormal PT, PTT, or both values corrected when calibration plasma (Instrumentation Laboratories) was added in a 50:50 ratio to patient plasma. Calibration plasma is obtained from healthy donors and is processed to maintain the characteristics of normal plasma. Normalization by calibration plasma indicates one or more factor deficiencies as the cause of the PT or PTT prolongation (as opposed to circulating anticoagulants such as heparin). In one patient these values did not normalize, which was interpreted as heparin contamination; this patient was excluded for evaluation of PT and PTT.

There were no correlations between the liver-function abnormalities and factor abnormalities.

The comparison between coagulation factors in infants before the BDG procedure and healthy control subjects is summarized in Table 5. Significant reductions in both pro-
TABLE 3. Normal ranges for coagulation factors and serum protein levels, as established from control subjects

\begin{tabular}{lrrc}
\hline Variable & Median & Mean & Normal range* $^{*}$ \\
\hline Fibrinogen (mg/L) & 268 & 278 & $178-555$ \\
Factor II & 89 & 90 & $69-122$ \\
Factor V & 115 & 117 & $84-152$ \\
Factor VII & 86 & 89 & $36-142$ \\
Factor VIII & 77 & 77 & $40-130$ \\
Factor IX & 66 & 66 & $41-104$ \\
Factor X & 92 & 94 & $72-134$ \\
Antithrombin III & 108 & 106 & $67-132$ \\
Plasminogen & 90 & 89 & $60-120$ \\
Protein C & 84 & 84 & $50-134$ \\
Protein S & 83 & 88 & $45-135$ \\
\hline
\end{tabular}

All factors except fibrinogen are expressed as percentage of activity. *Normal ranges were based on empirical $95 \%$ confidence intervals derived from the 2.5 and 97.5 percentile limits ( $n=34$, except fibrinogen: $n$ $=16)$.

coagulant and anticoagulant factor levels were detected before the BDG in all 36 patients. The most common abnormalities were low levels of factor II $(25 / 36)$, factor V (26/36), factor X (25/36), and protein C (23/36).

Univariate and multivariate logistic regression were used to examine the relationship of the 11 variables (ie, sex, weight, prior procedure, ventricle morphology [left or right], $\mathrm{EDP}, \mathrm{SvO}_{2}, \mathrm{Qp} / \mathrm{Qs}$ ratio, common atrial pressure, ventricular function, atrioventricular valve regurgitation, and serum albumin level) to specific coagulation factor abnormalities. For individual variables, a low serum albumin level was predictive of an abnormality in factor II, decreased ventricular function by echocardiography was predictive of an abnormality in factor VII, and a low $\mathrm{SvO}_{2}$ was predictive of an abnormality in factor $\mathrm{X}$ (all $P<.001$ ). The only significant predictor for multiple coagulation factor abnormalities was decreased ventricular function $(P<.001)$.

According to institutional practice, the cardiopulmonary bypass (CPB) circuit for all patients was primed with whole blood (WB) to achieve a hematocrit level of approximately $30 \%$. Patients received $2 \mathrm{mg} / \mathrm{kg}$ heparin before aortic cannulation and an additional $2 \mathrm{mg} / \mathrm{kg}$ in the circuit prime. The initial activated clotting time on CPB was greater than 500 seconds in all patients, despite the low antithrombin III level subsequently noted in $33 \%$ of the patients. Fifteen patients were transfused in the operating room with WB (mean volume, $163 \pm 93 \mathrm{~mL}$ ), 18 with packed red blood cells (mean volume, $144 \pm 100 \mathrm{~mL}$ ), 25 with platelets (mean volume, 69 $\pm 22 \mathrm{~mL}$ ), and 14 with cryoprecipitate (mean volume, $32 \pm$ $14 \mathrm{~mL}$ ); no patient received fresh frozen plasma, and 2 patients did not receive any blood products in the operating room but were transfused in the ICU postoperatively. An antifibrinolytic was administered intraoperatively to 16 
TABLE 4. The mean hematocrit, platelet count, coagulation, and liver function tests before the BDG

\begin{tabular}{|c|c|c|c|}
\hline & Pre-BDG & $\begin{array}{l}\text { No. of patients } \\
\text { outside normal } \\
\text { range }(\%)\end{array}$ & Normal range \\
\hline Hematocrit (\%) & $40.9 \pm 4.9$ & $1(3)$ & $33-55$ \\
\hline Platelet count $\left(\times 10^{3}\right)$ & $270 \pm 56$ & 0 & $130-400$ \\
\hline $\mathrm{PT}(\mathrm{s})$ & $13 \pm 0.8$ & $15(42)$ & $11.0-13.0$ \\
\hline $\mathrm{PTT}(\mathrm{s})$ & $56.9 \pm 16.5$ & $30(83)$ & $27-37$ \\
\hline Albumin (g/dL) & $3.4 \pm 0.4$ & $5(14)$ & $3.0-4.6$ \\
\hline AST (U/L) & $33.8 \pm 13$ & $6(16)$ & $2-40$ \\
\hline $\operatorname{ALT}(U / L)$ & $21.1 \pm 11.1$ & $3(8)$ & $0-35$ \\
\hline GGTP (U/L) & $14.7 \pm 9.7$ & 0 & $5-40$ \\
\hline ALK (U/L) & $277 \pm 124$ & $3(8)$ & $110-400$ \\
\hline Bilirubin (mmol/L) & $0.8 \pm 0.9$ & $5(14)$ & $0.3-1.2$ \\
\hline
\end{tabular}

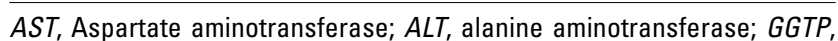
gamma-glutamyl transpepsidase; $A L K$, alkaline phosphatase.

patients (15 received traneximic acid and 1 received aprotinin), according to the surgeon's preference.

The mean chest tube drainage at 24 hours in the ICU was $146 \pm 82 \mathrm{~mL}$. Six patients received transfusion with WB (mean volume, $53 \pm 37 \mathrm{~mL}$ ) within 24 hours in the ICU, 25 patients received packed red blood cells (mean volume, 88 $\pm 29 \mathrm{~mL}$ ), 2 patients received cryoprecipitate (mean volume, $35 \pm 7 \mathrm{~mL}$ ), 5 patients received platelets (mean volume, $44 \pm 31 \mathrm{~mL}$ ), and 1 patient received fresh frozen plasma (30 mL).

Coagulation factors were assayed in the ICU 24 hours postoperatively. All factor levels were below the normal range when compared with those of our age-matched control subjects, except factor VIII and fibrinogen, which were significantly higher. When pre- and post-BDG levels were compared within the same patients, fibrinogen and factor VIII and IX values were significantly higher than preoperative values (all $P<.001$ ), and in addition, factor VII and protein $\mathrm{S}$ values were significantly lower than preoperative values $(P<.001$ for each).

One patient with hypoplastic left heart syndrome who had previously undergone a Norwood procedure had a large clot in the common atrium on postoperative day 2, with subsequent low cardiac output requiring extracorporeal membrane oxygenation. Although the patient was taken back to the operating room for evacuation of the thrombus, she subsequently died on postoperative day 3 . All coagulation factors in this patient before the BDG procedure were in the low normal range, except for protein $\mathrm{C}$, which was substantially below the norm for age (39\%). The immediate postBDG factor levels in this patient were also notable for persistently low protein C level (35\%). This patient had not received an antifibrinolytic agent. All other patients recovered uneventfully without clinical sign of thrombus or problems with postoperative hemostasis.
TABLE 5. Mean factor levels between control subjects and patients immediately before the BDG procedure

\begin{tabular}{lrccc}
\hline Variable & $\begin{array}{r}\text { Control } \\
(\mathbf{n = 3 4 )}\end{array}$ & $\begin{array}{c}\text { Pre-BDG } \\
(\mathbf{n = 3 6 )}\end{array}$ & $\begin{array}{c}\boldsymbol{P}^{\text {value }} \\
\text { Fibrinogen (mg/L) }\end{array}$ & $\begin{array}{c}\text { No. (\%) of pre-BDG } \\
\text { patients below } \\
\text { normal range }\end{array}$ \\
\hline Factor II (\%) & $90 \pm 89$ & $187 \pm 51$ & $<.001$ & $16(44)$ \\
Factor V (\%) & $117 \pm 18$ & $76 \pm 12$ & $<.001$ & $25(69)$ \\
Factor VII (\%) & $89 \pm 20$ & $45 \pm 12$ & $<.001$ & $26(72)$ \\
Factor VIII (\%) & $77 \pm 19$ & $65 \pm 22$ & .001 & $7(19)$ \\
Factor IX (\%) & $66 \pm 14$ & $42 \pm 9$ & $<.001$ & $17(47)$ \\
Factor X (\%) & $94 \pm 13$ & $69 \pm 12$ & $<.001$ & $25(69)$ \\
Antithrombin III (\%) & $106 \pm 12$ & $70 \pm 11$ & $<.001$ & $12(33)$ \\
Plasminogen (\%) & $89 \pm 14$ & $70 \pm 17$ & $<.001$ & $12(33)$ \\
Protein C (\%) & $84 \pm 18$ & $45 \pm 15$ & $<.001$ & $23(64)$ \\
Protein S (\%) & $88 \pm 19$ & $73 \pm 20$ & .002 & $2(6)$
\end{tabular}

Data are presented as mean percentage $\pm S D$, except for fibrinogen level (fibrinogen, $\mathrm{n}=18$ for control subjects; protein $\mathrm{S}, \mathrm{n}=31$ for pre-BDG patients).

${ }^{*}$ Based on 2-sample Student $t$ tests.

\section{Discussion}

Our study found that when compared with healthy infants of similar age, there was a high incidence of both procoagulant and anticoagulant factor abnormalities in patients with single-ventricle physiology before the cavopulmonary connection. Thus, we conclude that significant abnormalities in multiple coagulation factors may be present before Fontan (and BDG) operations.

The finding of coagulation factor abnormalities in children with single-ventricle physiology has been previously reported after the Fontan operation. Cromme-Dijkhuis and colleagues ${ }^{9}$ examined 37 patients for coagulation factor abnormalities to determine whether prothrombotic risk factors could explain the increased incidence of thromboembolism observed after the Fontan procedure. The most pronounced abnormality was a deficiency of protein $\mathrm{C}$; additionally, the concentrations of antithrombin III and factors II and X were significantly lower in protein $\mathrm{C}$-deficient patients than in those with normal protein $\mathrm{C}$ concentrations. This study suggested that the high thrombotic risk after the Fontan operation could be caused by, or at least associated with, an imbalance between procoagulant and anticoagulant factors that favored thrombus formation and that one or more aspects of Fontan physiology were responsible; similar results and conclusions have been reported by others. ${ }^{6,10,11}$ However, these studies did not examine the full complement of coagulation factors, did not use agematched control subjects, and did not study patients earlier in their course of single-ventricle physiology.

The hemostatic system matures over the first several years of life, ${ }^{14,15}$ leading to important differences in factor 
and activity measurements in children compared with those in adults; thus, use of age-matched control subjects is essential. In general, values of most procoagulant and anticoagulant factors are lowest in neonates and infants and increase toward adult values at varying rates. One study of 246 children aged 1 to 16 years demonstrated that mean values of 7 procoagulants were significantly lower than adult values, increasing with age. Values for 3 circulating inhibitors also differed from adult values, with protein $\mathrm{C}$ in particular being significantly lower compared with adult values. ${ }^{14}$ Despite the observed differences, there is no evidence that infants are at greater risk for hemorrhagic or thrombotic problems compared with adults, suggesting that the neonatal and infant system is in functional balance at lower concentrations of most factors. ${ }^{17}$

Although many of the coagulation factor levels in our patients before the BDG procedure were low, postoperative bleeding did not appear to be a problem. This could indicate that the coagulation systems in these infants are indeed balanced, which is consistent with the prior observation of functional maturity of the coagulation system in neonates and infants. ${ }^{17}$ However, whether the coagulation factor abnormalities (compared with those of age-matched control subjects) we measured in our patients are due to delayed maturation caused by cyanosis or low cardiac output is speculative. Severe hepatic synthetic dysfunction (eg, reduced albumin levels) or evidence of hepatocellular dysfunction (eg, bilirubin or transaminase) did not appear to be associated with coagulation abnormalities, an important observation given the prominent role of the liver in the synthesis of procoagulants and anticoagulants and the potential for altered liver blood flow and oxygen delivery in these patients. Patients with decreased ventricular function were more likely to demonstrate multiple factor deficiencies; however, we were unable to relate specific hemodynamic variables that could contribute to hepatic congestion or altered hepatic synthetic function with specific procoagulant or anticoagulant abnormalities. This may in part be due to the fact that significant hemodynamic abnormalities were uncommon, which also suggests that the observed factor abnormalities are unrelated in this group of patients.

Only 5 of our patients had not undergone a surgical procedure before the BDG operation; nevertheless, there were no differences in coagulation factor abnormalities in this small group of patients when compared with patients who had undergone prior surgery (ie, stage 1 procedure, pulmonary artery band, or Blalock-Taussig shunt). It is unknown whether children with congenital heart disease per se have procoagulant and anticoagulant abnormalities. A comparison with healthy age-matched control subjects and measurement of factor levels in nonhypoxic infants with 2ventricle physiology congenital heart defects would be necessary to answer this question.
Coagulation factor abnormalities 24 hours after CPB are difficult to evaluate because of changes in the plasma concentration of coagulation proteins from hemodilution during $\mathrm{CPB}$, consumption coagulopathy, the acute-phase response, and transfusion of blood products, including cryoprecipitate, platelets, and WB. In particular, immediate postoperative increases in the concentrations of fibrinogen and factor VIII, as reported here, are difficult to interpret because they are well-described acute-phase reactants. ${ }^{18,19}$ Whether changes in these 2 variables represent an acute-phase reaction or reflect the development of a hypercoagulable state induced by the BDG procedure, as has been previously concluded for the Fontan operation, ${ }^{11}$ cannot be determined. Post-BDG patients had elevated levels of factor II compared with that seen in pre-BDG patients. This, combined with low levels of antithrombin III, could increase the risk for postoperative thrombosis because the ratio of factor II and antithrombin III levels has been shown to be more important than protein $\mathrm{C}$ and $\mathrm{S}$ levels in terms of thrombotic risk. ${ }^{20}$ Whether the potential prothrombotic state described here in the postoperative period persists beyond the immediate postoperative state in these patients is as yet undetermined.

Previous studies, examining only a limited number of clotting factors at one point in time and not using agematched control subjects, suggested that procoagulant and anticoagulant factor abnormalities are the result of the Fontan operation and physiology. They further concluded that the increased risk of stroke and other thromboembolic events is the direct outcome of these abnormalities. The inherent limitations in these conclusions have been emphasized recently. ${ }^{12,13}$ On the basis of the present study, it appears that multiple procoagulant and anticoagulant factor abnormalities occur early in the course of single-ventricle physiology and in fact precede the cavopulmonary connection. Whether these abnormalities are part of a genetic predisposition or result from other hemodynamic or pathophysiologic abnormalities and what their contribution might be to deleterious clinical events require further study. A prospective, longitudinal evaluation of the changes in coagulation status in a cohort of patients with the same diagnosis undergoing staged single-ventricle repair is currently underway and may help determine the time course of coagulation abnormalities in these patients.

\section{References}

1. Fontan F, Baudet E. Surgical repair of tricuspid atresia. Thorax. 1971;26:240-8.

2. Stamm C, Friehs I, Mayer JE, Zurakowski D, Triedman JK, Moran AM, et al. Long-term results of the lateral tunnel Fontan operation. $J$ Thorac Cardiovasc Surg. 2001;121:28-41

3. Mayer JE Jr, Bridges ND, Lock JE, Hanley FL, Jonas RA, Castaneda AR. Factors associated with marked reduction in mortality for Fontan operations in patients with single ventricle. J Thorac Cardiovasc Surg. 1992;103:444-52.

4. Cromme-Dijkhuis AH, Hess J, Hahlen K, Henkens CM, Bink-Boelkens MT, Eygelaar AA, et al. Specific sequelae after Fontan operation at mid- 
and long-term follow-up. Arrhythmia, liver dysfunction, and coagulation disorders. J Thorac Cardiovasc Surg. 1993;106:1126-32.

5. Rosenthal DN, Friedman AH, Kleinman CS, Kopf GS, Rosenfeld LE, Hellenbrand WE. Thromboembolic complications after Fontan operations. Circulation. 1995;92(Suppl):II-287-93.

6. Jahangiri M, Ross DB, Redington AN, Lincoln C, Shinebourne EA. Thromboembolism after the Fontan procedure and its modifications. Ann Thorac Surg. 1994;58:1409-14.

7. Balling G, Vogt M, Kaemmerer H, Eicken A, Meisner H, Hess J. Intracardiac thrombus formation after the Fontan operation. J Thorac Cardiovasc Surg. 2000;119:745-52.

8. Jahangiri M, Shore D, Kakkar V, Lincoln C, Shinebourne E. Coagulation factor abnormalities after the Fontan procedure and its modifications. J Thorac Cardiovasc Surg. 1997;113:989-93.

9. Cromme-Dijkhuis AH, Henkens CM, Bijleveld CM, Hillege HL, Bom VJ, van der Meer J. Coagulation factor abnormalities as possible thrombotic risk factors after Fontan operations. Lancet. 1990;336:1087-90.

10. van Nieuwenhuizen RC, Peters M, Lubbers LJ, Trip MD, Tijssen JG, Mulder BJ. Abnormalities in liver function and coagulation profile following the Fontan procedure. Heart. 1999;82:40-6.

11. Jahangiri M, Kreutzer J, Zurakowski D, Bacha E, Jonas RA. Evaluation of hemostatic and coagulation factor abnormalities in patients undergoing the Fontan operation. J Thorac Cardiovasc Surg. 2000;120:778-82.

12. Monagle P, Andrew M. Coagulation abnormalities after Fontan procedures [letter]. J Thorac Cardiovasc Surg. 1998;115:732-3.
13. Sluysmans T, Ovaert C, d'Udekem Y, Barrea C. Coagulation factor abnormalities after the Fontan procedure and its modifications [letter]. J Thorac Cardiovasc Surg. 1999;117:1038.

14. Andrew M, Vegh P, Johnston M, Bowker J, Ofosu F, Mitchell L. Maturation of the hemostatic system during childhood. Blood. 1992;80:1998-2005.

15. Andrew M, Paes B, Milner R, Johnston M, Mitchell L, Tollefsen DM, et al. Development of the human coagulation system in the full-term infant. Blood. 1987;70:165-72.

16. Wright EM, Royston P. Calculating reference intervals for laboratory measurements. Stat Methods Med Res. 1999; 8:93-112.

17. Miller BE, Bailey JM, Mancuso TJ, Weinstein MS, Holbrook GW, Silvey EM, et al. Functional maturity of the coagulation system in children: an evaluation using thrombelastography. Anesth Analg. 1997;84:745-8.

18. Chan AK, Leaker M, Burrows FA, Williams WG, Gruenwald CE, Whyte L, et al. Coagulation and fibrinolytic profile of paediatric patients undergoing cardiopulmonary bypass. Thromb Haemost. 1997;77:270-7.

19. O'Donnell J, Tuddenham EG, Manning R, Kemball-Cook G, Johnson D, Laffan M. High prevalence of elevated factor VIII levels in patients referred for thrombophilia screening: role of increased synthesis and relationship to the acute phase reaction. Thromb Haemost. 1997;77:825-8.

20. Butenas S, van't Veer C, Mann KG. "Normal" thrombin generation. Blood. 1999;94:2169-78.

\section{Targeted}

The Journal of Thoracic and Cardiovascular Surgery gives you two tables of contents.

The condensed table of contents tells you at a glance what topics and authors are presented each month. The expanded table of contents gives you a brief abstract of each article. You select only those articles of most interest to you for further reading. 